\title{
WEDGES IN ABELIAN GROUPS AND IN LINEAR TOPOLOGICAL SPACES
}

\author{
BY \\ J. G. MACCARTHY
}

1. Introduction. In an abelian group a subset $S$ having the property that the family of all its "translates" is closed under finite intersection will be called a "wedge" (see Definition 2). Wedges with associated topological or order properties appear in the literature as " $C$-cones," "minihedral" cones, etc. Clarkson in [1] used this notion of wedge to characterize Banach spaces of functions continuous on compact Hausdorff domains. Fullerton in [2] shows that the wedge property implies several of the properties which were independently postulated in [1]. In [3] the concept of wedge is used to characterize Banach spaces of integrable functions. The word "wedge" with a restricted meaning appears in [5]. See also [4].

In this paper we show that in a linear space over a zero-characteristic field of scalars a subset is a wedge iff it is an ideal in some lattice-ordering of the additive group of the space. Then we show how this equivalence can shorten the proof of the theorem in [2] while in fact proving slightly more. But for the most part it is in the broader setting of abelian groups (rather than linear topological spaces) that we exhibit properties of the wedge. In particular we show that every lattice-ideal (not merely principal lattice-ideal) in an abelian group is a wedge. The converse question (whether in a torsionfree abelian group, $G$, any wedge is an ideal in some lattice-ordering of $G$ ) is not given an unqualified answer here. However for divisible torsion-free abelian groups the equivalence of "ideal" and "wedge" is established in this paper-thus leading to the theorem mentioned in the first sentence of this paragraph.

In the course of the argument it is also shown how the wedge concept may be used to partition the family of all abelian groups into the familiar "torsion," "mixed" and "torsion-free" subclasses. Also the existence of certain everywhere-dense wedges in the additive group of the reals is established and all possible wedges in the additive group of the rationals are constructed.

2. Conventions. All groups referred to here are abelian and will be denoted by " $(G,+)$ " or briefly, " $G$." A natural homomorphism $G \rightarrow G / F$ will always be denoted by " $h$."

Definition 1. If $A$ and $B$ are subsets of $(G,+)$ then " $A+B$ " shall denote $\{a+b \mid a$ in $A, b$ in $B\}$. If $B$ is singleton $\{b\}$ we write " $A+b$."

Definition 2. $K$ will be called a wedge in $G$ if $K$ is a nonempty proper subset of $G$ such that for any $a, b$ in $G$ there exists $c$ in $G$ satisfying the settheoretic equation

Received by the editors November 15,1960 . 


$$
(K+a) \cap(K+b)=(K+c) .
$$

Definition 3. If $A \subseteq G$ then by the "Fixgroup," $F$, of $A$ we mean the set $\{f$ in $G \mid A+f=A\}$.

Definition 4. A nonempty abelian group is a "torsion group" iff it is not the null-group, $\{0\}$, and every element is of finite order, "torsion-free" iff its only element of finite order is the identity, "mixed" if neither a torsion group nor torsion-free.

Definition 5. A wedge will be called "free" iff its Fixgroup is the nullgroup. Otherwise it will be called "fixed."

Definition 6. A wedge will be called regular iff its Fixgroup is torsionfree. Otherwise a wedge is "irregular."

Definition 7. $G$ is said to be lattice-ordered (an $l$-group) iff its elements are lattice-ordered "homogeneously"-that is, for any $a, b, x$ in $G$

$$
a \leqq b \Leftrightarrow a+x \leqq b+x .
$$

Definition 8. A $u$-ideal in a lattice $L$ is a subset of $L$ such that for any $a, b$ in $L$

$$
a, b \text { in } I \Leftrightarrow a \cup b \text { in } I \text {. }
$$

Or, equivalently, $I$ is a $u$-ideal in the lattice $L$ if and only if

(1) $a, b$ in $I \Rightarrow a \cup b$ in $I$ and also

(2) $a$ in $I, b$ in $L \Rightarrow a n b$ in $I$.

A $n$-ideal is the dual.

Finally, frequent reference will be made to the principle " $P$ ":

"If $A$ and $B$ are sets and if $f^{-1}$ is single-valued on $f(A \cup B)$ then $f(A \cap B)$ $=f(A) \cap f(B) . "$

3. Wedges everywhere-dense on the real line. The obvious wedge in the additive group of the reals, $R$, is the "half-line" (closed or open) relative to the usual ordering. We show that there exist other wedges in $R$.

THEOREM 1. Let $m$ be an automorphism of $G$ onto $G$. Let $K$ be a wedge in $G$. Then $m[K]$, the automorphic image of $K$, is a wedge in $G$.

Proof. For any $a, b$ in $G$,

$$
\begin{aligned}
(m[K]+a) \cap(m[K]+b) & =m\left(K+m^{-1}[a]\right) \cap m\left(K+m^{-1}[b]\right) \\
& =m\left\{\left(K+m^{-1}[a]\right) \cap\left(K+m^{-1}[b]\right)\right\}, \text { by "P"; } \\
& =m[K+c], c \text { in } G \\
& =m[K]+m[c] .
\end{aligned}
$$


COROLlaRy 1.1. There exists in $R$ an everywhere-dense wedge $K$ with everywhere-dense complement $K^{\prime}$ which is also a wedge. (Let $K$ be a discontinuous automorphic image of the wedge of positives. It is well known that any discontinuous map of one Hamel basis onto another defines such an automorphism, always mapping an interval onto an everywhere-dense image.)

This corollary may easily be generalized to other linear topological spaces. But not to all, for it will be shown in Theorem 11 that there are no such "pathological" wedges in the additive group of the rationals. In order to prove Theorem 11 we first establish some generalities as follows.

4. Wedges as lattice-ideals. The next theorem is due to M. H. Stone. It is proven, without the assumption of commutativity, in [6].

THEOREM 2. Let $\nabla$ be a binary operation over $G$ satisfying, for any $a, b, c$ in $G$, the four conditions:

1. $a \nabla a=a$,

2. $a \nabla b=b \nabla a$,

3. $(a \nabla b) \nabla c=a \nabla(b \nabla c)$,

4. $a+(b \nabla c)=(a+b) \nabla(a+c)$.

Then $G$ is a lattice-ordered group with respect to the operation $\nabla$. (That is: the relation " $\leqq$ " defined by the equivalences $a \leqq b \Leftrightarrow a \nabla b=a \Leftrightarrow-a \nabla-b=-b$, lattice-orders $G$ in the sense of Definition 7.)

It is known [1] that a free wedge in a linear topological space induces a lattice ordering of the space; in the following theorem it is shown how a more general result concerning the ordering effect of a free wedge may be inferred at once from Theorem 2 .

Theorem 3. Suppose $K$ is a free wedge in $G$. Then

1. $G$ is non-null torsion-free.

2. $K$ induces in $G$ a distributive lattice-ordering, $L$, under which $G$ is a lattice-ordered group.

3. $K$ is a proper ideal in $G$ with respect to $L$.

Proof of 2. Define in $G$ an operation $\boldsymbol{n}$ by the convention

$$
a \cap b=c \text { iff }(K+a) \cap(K+b)=K+c .
$$

Then $\boldsymbol{n}$ satisfies the conditions of Theorem 2. (For example, condition 4 is fulfilled since $a+[(K+b) \cap(K+c)]=(K+a+b) \cap(K+a+c)$ by " $P$ " if we interpret addition of " $a$ " as the mapping $f$ ). The equivalent relation "ミ" on $G$ is then defined by

$$
a \leqq b \Leftrightarrow a \cap b=a \Leftrightarrow(K+a) \cap(K+b)=K+a \Leftrightarrow K+a \subseteq K+b .
$$

Finally, any $l$-group is a distributive lattice [6].

Proof of 3. By $2, K$ induces a lattice-ordering, $L$, in $G$. With respect to 
$L$, for any $a, b$ in $K$ set $a \cap b=p$ and with no loss of generality (because of the homogeneity of $L$ ) take $p$ to be 0 . Then

$$
a \cap b=0 \Leftrightarrow(K+a) \cap(K+b)=K .
$$

By the Dedekind Law for abelian $l$-groups $a+b=a \cup b+a \cap b$; hence $a+b$ $=a \cup b$. But $b$ in $K \Rightarrow a+b$ in $K+a$ and $a$ in $K \Rightarrow a+b$ in $K+b$ and so $a$ u $b$ $=a+b$ is in $(K+a) \cap(K+b)=K$. Thus condition 1 of Definition 8 is satisfied. Regarding condition 2 , for any $a$ in $K$ and $b$ in $G$

$$
a \cap b \leqq a \text { iff }(K+a n b) \subseteq K+a .
$$

Setting $a=0$ this becomes

$$
0 \cap b \leqq 0 \text { iff }(K+0 \cap b) \subseteq K .
$$

But $a(=0)$ in $K$ implies

$$
0 \cap b \text { in } K+0 \cap b .
$$

This with (1) implies

$$
0 \cap b(=a \cap b) \text { in } K(=K+a) .
$$

Hence condition 2 of Definition 8 is satisfied and $K$ is an ideal with respect to $L$ and in particular is a $u$-ideal. Since by definition $K$ is a proper subset of $G, K$ is a proper $u$-ideal.

Proof of 1 . It is known that any $l$-group is torsion-free [6]. Also, since $K$ is a proper subset of $G, G$ is non-null.

Corollary 3.1. By the definition of $L$ in the preceding theorem, the family of all "translates" $K+x, x$ in $G$, of a free wedge $K$ is distributively latticeordered (by the inclusion relation $\subseteq$ defined over the subsets of $G$ ) lattice-isomorphically to $L$ defined on the points of $G$.

The last theorem has shown that a free wedge is an ideal. That not all fixed wedges are ideals is implied below by Theorem 10 . The next two theorems are concerned with the converse question.

Theorem 4. Any proper principal ideal I in an l-group $G$ is a free wedge.

Proof. Since $I$ is proper, $G$ is not the null-group. That $I$ is a wedge follows at once from the homogeneity of the lattice-ordering. For, without loss of generality taking $I$ to be the principal ideal of "positives": $\{x \mid 0 \leqq x\}$, then for any $y$ in $G, I+y$ is the set $\{x \mid y \leqq x\}$ and hence $I \cap(I+y)=I+0 \cup y$. That the wedge is free follows from the implications:

$$
\{x \mid y \leqq x\}=\{x \mid 0 \leqq x\} \Leftrightarrow 0 \leqq y \text { and } y \leqq 0 \Leftrightarrow 0=y .
$$

Dropping the restriction that the ideal $K$ be principal, we have the more general result: 
THEOREм $5\left({ }^{1}\right)$. Let $L$ be a lattice ordering of $G$ and let $K$, with respect to $L$, be a proper $\mathrm{U}$-ideal in $G$. Then $K$ is a wedge and for any $a, b$ in $G$

$$
(K+a) \cap(K+b)=K+a \cap b .
$$

Proof. $x$ in $K+a n b \Leftrightarrow$, for some $k$ in $K, x=k+a \cap b \leqq k+a \Leftrightarrow x-a$ $\leqq k \Rightarrow x-a$ in $K \Leftrightarrow x$ in $K+a$. Similarly $x$ in $K+b$. Next, $x$ in $(K+a)$ $\cap(K+b) \Leftrightarrow$, for some $k_{1}, k_{2}$ in $K, x=k_{1}+a=k_{2}+b \Rightarrow a n b=\left(x-k_{1}\right) \cap\left(x-k_{2}\right)$ $=x+\left(-k_{1}\right) \cap\left(-k_{2}\right)=x-k_{1} \cup k_{2} \Rightarrow x=k_{1} \cup k_{2}+a \cap b$ in $K+a \cap b$. (The dual statement for a $\mathrm{n}$-ideal would read $(K+a) \cap(K+b)=K+a \cup b$.) Theorens 3 and 4 together imply

THEOREM 6. G contains a free wedge iff $G$ is non-null and torsion-free.

Proof. The "only if" is part of Theorem 3. The other half follows from the fact that any torsion-free abelian group is lattice-orderable and thus contains a principal ideal of positives which is proper if $G$ is non-null and is hence, by Theorem 4, a free wedge.

5. Homomorphisms modulo subgroups of the Fixgroup. For the discussion of fixed wedges the following lemmas and theorems concerning natural homomorphic images of wedges are introduced. Most of the proofs in this section are left to the reader. Essentially the idea here is to show that the mapping principle " $P$ " is still applicable even when the mapping $f$ is an homomorphism, with multiple-valued inverse, if the kernel of the homomorphism satisfies an appropriate condition.

Lemma 1. Let $A$ be a subset of $G, F$ the Fixgroup of $A$ and $P$ any subgroup of $G$. Then $P \subseteq F$ iff $A$ is a union of cosets of $P$.

From this lemma there follows at once

Lemma 2. Suppose $A \subseteq G$ and that $P$ is a subgroup of the Fixgroup of $A$ and that $h$ is the natural homomorphism $G \rightarrow G / P$. Then $h^{-1}[h(A)]=A$.

Lemмa 3. Suppose $A \subseteq G$ and $B \subseteq G$. Suppose that $F$ and $H$ are respectively the Fixgroups of $A$ and $B$ and that $P$ is a subgroup of $F \cap H$. Let $h$ denote the natural homomorphism $G \rightarrow G / P$. Then $h(A) \cap h(B)=h(A \cap B)$.

Proof.

$$
\begin{aligned}
h(A) \cap h(B) & =h\left\{h^{-1}[h(A) \cap h(B)]\right\} \\
& =\left(\text { by }^{\prime \prime} P^{\prime \prime}\right) h\left\{\left[h^{-1} h(A)\right] \cap\left[h^{-1} h(B)\right]\right\} \\
& =(\text { by Lemma } 2) h\{A \cap B\} .
\end{aligned}
$$

(1) The author is indebted to the referee for this abbreviation of the author's original proof. 
Lemma 4. Let $A$ be a subset of $G$ with Fixgroup $F ; P, a$ subgroup of $F ; h$, the natural homomorphism $G \rightarrow G / P$. Then $h(F)$ is the Fixgroup of $h(A)$.

LEMMA 5. Let $h$ be the natural homomorphism $G \rightarrow G / P$ where $P$ is any subgroup of $G$. If $A \subseteq G / P$ and if $X$ is the Fixgroup of $A$ in $G / P$ then $h^{-1}(X)$ is the Fixgroup of $h^{-1}(A)$ in $G$. lish

Using Lemmas 3 and 4, the proof of Theorem 1 may be imitated to estab-

Theorem 7. Let $K$ be a wedge in $G$ with Fixgroup $F$. Let $P$ be a subgroup of $F$ and let $h$ denote the natural homomorphism $G \rightarrow G / P$. Then $h(K)$ is a wedge in $G / P$ with Fixgroup $h(F)$.

From this and Lemma 5 we next infer

TheOREM 8. Let $h$ denote the natural homomorphism $G \rightarrow G / P$ where $P$ is any subgroup of $G$. A subset $K$ of $G / P$ is a free wedge in $G / P$ iff $h^{-1}(K)$ is a wedge in $G$ having the Fixgroup $P$.

Also, from Lemma 3 together with Theorems 3 and 5 we can infer

TheOREм 9. Let $K$ be an ideal with respect to a lattice ordering $L$ of $G$. Let $F$ be the Fixgroup of $K$. Let $h$ denote the natural homomorphism $G \rightarrow G / F$. Let $B$ denote the lattice ordering of $G / F$ induced by $h(K)$. Then $h$ is a lattice-homomorphism of $L$ onto $B$.

6. Aperiodicity of wedges. All wedges are "aperiodic" in the sense that for any positive integer $n$ and any nonzero $x$ in $G$,

$$
K+n x=K \Leftrightarrow K=K+x \text {, where } K \text { is a wedge in } G .
$$

This property, superficially reasonable looking in a lattice-ordered (and hence torsion-free) $G$, pertains to a wedge in any group, as will now be shown. As a consequence, it is impossible for a torsion group to contain any wedge. A more general consequence appears below as Theorem 10 .

Definition 9. A subgroup $P$ of $G$ will be called "pure" iff $x$ in $G, n x$ in $P \Rightarrow x$ in $P$ whenever $n$ is a nonzero integer. The following lemma is immediately clear.

Lemma 6. $G / F$ is torsion-free iff $F$ is pure.

Lemмa 7. $F$ is the Fixgroup of a wedge $K$ in $G$ iff $G / F$ is non-null and torsion-free.

Proof. If $F$ is the Fixgroup of $K$ then $h(K)$ is by Theorem 7 a free wedge in $G / F$ and this implies by Theorem 6 that $G / F$ is non-null and torsion-free. Conversely, if $G / F$ is non-null and torsion-free then, by Theorem $6, G / F$ contains a free wedge, $V$, and, by Theorem $8, h^{-1}(V)$ is a wedge in $G$ having Fixgroup $F$. 
Combining Lemmas 6 and 7 and noting that $G / F$ is non-null iff $F$ is a proper subgroup of $G$, we infer:

LEMMA 8. $F$ is the Fixgroup of a wedge in $G$ iff $F$ is a pure and proper subgroup of $G$.

Lemma 9. Denoting by " $M$ " the maximal torsion subgroup of $G$, if $F$ is the Fixgroup of a wedge in $G$ then $M \subseteq F$.

Proof. Noting that $M$ is itself pure, it is easy to show that $M$ is in fact the intersection of all pure subgroups of $G$. Hence, by Lemma $8, M \subseteq F$.

THEOREM 10. If $G$ is a non-null abelian group then

(a) $G$ contains a regular wedge iff $G$ is torsion-free.

(b) $G$ contains an irregular wedge iff $G$ is mixed.

(c) $G$ contains no wedge iff $G$ is a torsion group.

Proof of (a). If $G$ contains a regular wedge, $K$, then the Fixgroup, $F$, of $K$ is (by the regularity) torsion-free; hence, by Lemma $9, M$ is the null-group and thus $G$ is torsion-free. Conversely, if $G$ is torsion-free, setting $F=\{0\}$ in Lemma 7 the lemma implies that $G$ contains a free wedge; any free wedge is regular.

Proof of (c). Since a wedge, $K$, is by definition properly included in its group, $G$, it follows that the Fixgroup, $F$, of $K$ is also properly included in $G$. But then Lemma 9 implies that $M$ must be a proper subset of $G$. Hence $G$ is not a torsion group if $G$ contains a wedge. Conversely, if $G$ is not a torsion group then $M$ is a proper subgroup of $G$; hence, since $M$ is also pure, Lemma 8 implies that $M$ is the Fixgroup of a wedge in $G$.

Proof of (b). By exclusion of the cases (a) and (c), this follows.

7. Wedges in the additive group of the rationals. As an application of the preceding theory, the question raised (just after Theorem 1) concerning the existence of "pathological" wedges in the additive group of the rationals (in analogy to those existing in the real line by Corollary 1.1) is answered negatively by

THEOREM 11. The only wedges in the additive group of the rationals, $R^{+}$, are the open or closed half-lines, $r>x$ or $r \geqq x$ and dually.

Proof. The proof depends upon the two following properties of $R^{+}$:

(a) The only pure subgroups of $R^{+}$are $R^{+}$and the null-group, $\{0\}$.

(b) The only possible lattice-ordering of $R^{+}$is the familiar linear ordering or its dual.

To establish (a), let $S$ be any non-null proper subgroup of $R^{+}$. Then $S$ contains a positive integer, $j$. Let $m / n$ lie in the complement of $S$. Then $(j n)(m / n)=j m$ is a nonzero integer-multiple of $m / n$ contained in $S$. Hence 
$S$ is not pure. That $R^{+}$and $\{0\}$ are pure is clear. To verify property (b), note that in any $l$-group, $G$, with a relation, " $\geqq$," the set of positives, $P:\left\{x \mid x^{\star} \geqq " 0\right\}$ is closed under multiplication by non-negative rationals, $r$, in the sense that $p$ in $P$ and $r p$ in $G$ imply $r p$ in $P$. Also, in any $l$-group, $x$ in $P$ and $-x$ in $P$ implies $x=0$. From this it is clear that the "positives" of $R^{+}$with respect to any lattice-ordering are either the familiar positives or their dual. But any homogeneous partial ordering of a group is uniquely determined by the set of its "positives." Hence, property (b) follows.

But Lemma 8 and property (a) imply that the Fixgroup of any wedge, $K$, in $R^{+}$is the null-group. Hence $K$ is a free wedge in $R^{+}$. By Theorem $3, K$ induces a lattice-ordering of $R^{+}$and $K$ is a proper ideal relative to this latticeordering. But by property (b) this lattice-ordering induced by $K$ must be the familiar linear ordering or its dual and the only ideals in this ordering are the half-lines.

The same argument may be used to show that the only wedges in the additive group of the integers are the half-lines, $j \leqq x$ or dually. But here the well-ordering permits much simpler approaches.

8. Equivalence of "wedge" and "lattice-ideal." Since only torsion-free groups are lattice-orderable we are concerned in this section only with regular wedges. By Theorem 5 every ideal is a wedge. A partial converse of Theorem 5 is given by Theorem 3; namely, that every free wedge is an ideal. The next two theorems concern the residual question, "Is every fixed regular wedge an ideal?"

Definition 10. Let $h: G \rightarrow G / F$ be a natural homomorphism of $G$. Let $B$ be a lattice-ordering of $G / F$. We shall say that $B$ can be "lifted" (from $G / F$ to $G$ ) iff there exists a lattice-ordering $L$ of $G$ such that $h$ is a lattice-homomorphism of $L$ onto $B$.

Theorem 12. Let $W$ be a wedge in $G$ and $F$ its Fixgroup. Let $h$ be the natural homomorphism $G \rightarrow G / F$. Then the lattice-ordering, $B$, of $G / F$ induced by $h(W)$ can be lifted to a lattice-ordering, $L$, of $G$ iff $W$ is an ideal with respect to $L$.

Proof. The set $h(W)$ is a free wedge in $G / F$, by Theorem 7 , and by Theorem 3 is an ideal with respect to $B$. If $B$ can be lifted to $L$ then $h$ is a latticehomomorphism. But then the pre-image of an ideal with respect to $B$ must be an ideal with respect to $L$. That is, $h^{-1} h(W)=W$ (by Lemma 2) is an ideal with respect to $L$. The converse statement that, if $W$ is an ideal in $G$ with respect to $L$ then the lattice-ordering $B$, induced by $h(W)$, can be lifted to $L$ is Theorem 9.

Theorem 12 implies (we omit the details) the equivalence of the statements:

(1) Every wedge in a torsion-free $G$ is an ideal.

(2) If $G$ is torsion-free then every lattice-ordering, $B$, of a non-null factorgroup $G / F$ can be lifted to $G$. 
Although the hypothesis of divisibility is a drastic one, the following theorem leads naturally to the consideration of linear spaces.

THEOREM 13. Let $G$ be a divisible torsion-free abelian group and h a natural homomorphism $G \rightarrow G / F$. Then any lattice ordering, $B$, of $G / F$ can be lifted to $G$.

Proof. $G / F$ lattice orderable with ordering, $B, \Leftrightarrow G / F$ torsion-free

$$
\begin{aligned}
& \Leftrightarrow F \text { is pure (by Lemma } 6 \text { ) } \\
& \Leftrightarrow F \text { is a direct summand of } G \\
& \Leftrightarrow G=F \oplus G / F \text {, where " } \oplus \text { " denotes "direct sum." }
\end{aligned}
$$

Since $F$ is torsion-free and abelian it is lattice-orderable. Let $R$ denote a lattice-ordering of $F$. Then the cardinal product $R \times B$ is a homogeneous lattice-ordering of $G$ which is a "lifting" of $B$.

9. Wedges in linear spaces. Since the additive structure of a linear space with scalars in a field of characteristic zero is a divisible torsion-free abelian group, Theorems 12 and 13 imply the following

THEOREM 14. In a linear space, L, having a zero-characteristic field of scalars a subset $S$ is a wedge iff it is an ideal in some appropriate lattice-ordering of the additive group of $L$. The number of possible lattice-ideal structures of $S$ is at least as great as the number of possible lattice-orderings of the Fixgroup, $F$, of $S$. (Since, if 0 in $S, S+F=S \Rightarrow F \subseteq S$-and thus to each new lattice-ordering, $R$, of $F$ in the proof of Theorem 13 there corresponds at least one new lattice-ideal structure for $S$.)

For the next theorem three definitions are needed.

Definition 11. By the "linear topology" for a linear space is meant the topology arising from the convergence scheme: if $a_{n}=b_{n} x+c_{n} y, x \neq y, b_{n}+c_{n}$ $=1, b_{n} \rightarrow b, c_{n} \rightarrow c$ then $a_{n} \rightarrow b x+c y$.

DEFinition 12. In a linear space over an ordered field a point, $p$, is an "extreme point" of $S$ if $p$ is in $S$ and $p$ is not between two other points of $S$.

DEFinition 13. A vector lattice is a lattice-ordered vector space over an ordered field, the lattice-ordering being invariant under both addition and multiplication, i.e., $x \leqq y \Leftrightarrow a+x \leqq a+y$ and $x \geqq 0 \Leftrightarrow k x \geqq 0$ for any non-negative scalar, $k$.

THEOREM 15. (a) If, in a linear space $S$ over the reals, $W$ is a wedge then $W$ is an ideal and conversely.

(b) If the wedge, $W$, has an extreme point, $p$, then

(b) $1: p$ is unique and $W$ is the principal ideal $\{x \mid x \geqq p\}$ in a uniquely defined lattice-ordering $L$ of $S$ and

(b) 2: the following five statements are equivalent. 
1. $S$ is a vector lattice with respect to $L$.

2. $S$ is a linear topological space in the order topology of $L$.

3. $W$ is closed in the linear topology of $S$.

4. $W$ is convex.

5. $W$ is a cone.

Proof. Part (a) follows from Theorem 14. To prove (b) 1 , take $W$ to be a u-ideal. Then for $x$ in $W, p=(1 / 2)(x \cap p)+(1 / 2)(p+[p-(x \cap p)])$. But $x \cap p$ is in $W$ and, since $p-(x \cap p) \geqq 0$, also $p+[p-(x \cap p)]$ is in $W$. Hence, if $p$ is an extreme point, $x \cap p=2 p-(x \cap p)$ which is equivalent to $x \geqq p$. This establishes (b)1. Finally the equivalences of (b) 2 are established as follows.

$1 \Rightarrow 2$ : A vector lattice is a linear topological space with respect to its order-topology because the mappings $x \rightarrow x+y, x \rightarrow k x(k>0)$ are lattice isomorphisms and are hence continuous in the variables $x$ and $y$ (separately and jointly) with respect to the order topology.

$2 \Rightarrow 3$ : First, the interval- order- and linear-topologies satisfy the respective inclusions $I \subseteq O \subseteq L$. For proof of the first inclusion see [7]. To prove the second, use subscripts $L$ and $O$ to indicate the linear and order topologies and we have, by definition of the linear topology,

$$
\underset{L}{\operatorname{Lim}}\left(a_{n} x+b_{n} y\right)=\left(\operatorname{Lim} a_{n}\right) x+\left(\operatorname{Lim} b_{n}\right) y
$$

and this, by the order-continuity of scalar multiplication, is equal to

$$
\operatorname{Lim}_{o}\left(a_{n} x\right)+\operatorname{Lim}_{o}\left(b_{n} y\right)
$$

and this, by the order-continuity of addition, equals

$$
\underset{o}{\operatorname{Lim}}\left(a_{n} x+b_{n} y\right) \text {. }
$$

But then the implication $2 \Rightarrow 3$ follows from the fact that the principal ideals of a lattice are a subbasis of closed sets for its interval-topology, hence $W$ is closed in $I$ and thus, by the above inclusions, also in $L$.

$3 \Rightarrow 4$ : Take $W$ to be the principal ideal of positives. The positives of an $l$-group are an additive semi-group which is closed (in the sense specified in the proof of Theorem 11) under non-negative rational multiplication. Thus if $x, y$ is in $W$ then $(1-s) x+s y$ is in $W$ for any rational $s$ in the unit interval and hence-since $W$ is closed in the linear topology-for any real $s$ in the unit interval. Therefore $W$ is convex.

$4 \Rightarrow 5$ : Again assume $W$ to be the ideal of positives and, noting once more the property of closure under non-negative rational multiplication, infer from this and the convexity that for any $x$ in $W$ and any positive rational, $s$, the pair $(\theta, s x)$ are end-points of a segment which is contained in $W$. Hence $W$ is a cone. 
$5 \Rightarrow 1$ : Conicity of the positives is equivalent to the "multiplicative homogeneity" property (Definition 13) of a vector lattice.

A proof of portions of part (b) of Theorem 15 appears in [2].

\section{REFERENCES}

1. James A. Clarkson, A characterization of C-spaces, Ann. of Math. vol. 48 (1947) pp. 845-850.

2. R. E. Fullerton, On a semi-group of subsets of a linear space, Proc. Amer. Math. Soc. vol. 1 (1950) pp. 440-442.

3. - A characterization of $L$-spaces, Fund. Math. vol. 38 (1951) pp. 127-136.

4. M. H. Stone, Algebraic formulation of the problem of measure, Acta Sci. Math. Szeged, vol. 12, Part B (1950) pp. 69-74.

5. Mahlon M. Day, Normed linear spaces, Berlin, Springer-Verlag, 1958.

6. Garrett Birkhoff, Lattice-ordered groups, Ann. of Math. vol. 43 (1942) pp. 298-331.

7. —, Lattice theory, Amer. Math. Soc. Colloquium Publications, vol. 25, 1948.

Greenbelt, Maryland 